\title{
R-CHOP intensification with mid-cycle methotrexate and consolidating AraC/TT with BCNU/aHSCT in primary aggressive lymphoma with CNS involvement
}

\author{
Maximilian J. Steinhardt ${ }^{1,4}$ (1) $\cdot$ Franziska C. Krummenast ${ }^{1} \cdot$ Andreas Rosenwald $^{2} \cdot$ Elena Gerhard-Hartmann $^{2}$. \\ Anke Heidemeier $^{3} \cdot$ Hermann Einsele $^{1} \cdot$ Max S. Topp $^{1} \cdot$ Johannes Duell ${ }^{1}$
}

Received: 9 May 2021 / Accepted: 11 May 2021 / Published online: 3 June 2021

(C) The Author(s) 2021

\begin{abstract}
Purpose Patients suffering from aggressive systemic peripheral lymphoma with primary central nervous system involvement (PCL) are a rare and sparsely investigated population. Recommended treatment regimens include a combination of intrathecal and systemic chemotherapy as well as whole brain radiotherapy while offering relatively poor survival.

Methods We conducted a single-center retrospective study that analyzed safety and outcome of $4+4$ cycles Rituximab (R)-CHOP and R-high-dose Methotrexate (HD-MTX) for newly diagnosed, transplant-eligible patients ("Ping-Pong"), followed by Cytarabine (AraC)/Thiotepa (TT), BCNU/TT, and autologous hematologic stem cell transplantation (aHSCT). We retrospectively analyzed a set of 16 patients with high-intermediate or high-risk IPI status.

Results Overall response rate to Ping-Pong was 100\% measured by CT/MRI, including $93.75 \%$ complete remissions after BCNU/TT followed by PBSCT. One patient failed to qualify for high-dose chemotherapy due to progression when receiving Cytarabine/TT. All patients experienced grade III adverse events, 3 of them a grade IV adverse event. Estimated progressionfree survival is $93.75 \%$ after a 4.8 -year follow-up currently.

Conclusion Our study suggests high effectivity of R-CHOP with mid-cycle MTX with aHSCT consolidation towards acceptable OS results in this challenging patient population.
\end{abstract}

Keywords Lymphoma $\cdot \mathrm{R}-\mathrm{CHOP} \cdot \mathrm{MTX} \cdot \mathrm{HD}$

\section{Introduction}

Johannes Duell

duell_j@ukw.de

Maximilian J. Steinhardt

steinhardt_m@ukw.de

1 Medizinische Klinik und Poliklinik II, Universitätsklinikum Würzburg, Oberdürrbacher Straße 6, 97080 Würzburg, Germany

2 Institut für Pathologie der Universität Würzburg, Universitätsklinikum Würzburg, Josef-Schneider-Straße 2, 97080 Würzburg, Germany

3 Institut für Diagnostische und Interventionelle Radiologie, Universitätsklinikum Würzburg, Oberdürrbacher Straße 6, 97080 Würzburg, Germany

4 Department of Internal Medicine II, University Hospital Würzburg, Oberdürrbacher Street 6, 97080 Würzburg, Germany
Diffuse large B-cell lymphoma (DLBCL) is the most common aggressive B-cell malignancy. Curative treatment is possible with R-CHOP and prognosis is evaluated on an age-adjusted IPI scoring system (aIPI). To this end, patients with an aIPI of zero have an excellent outcome of over $90 \%$ PFS at 5 years, whereas patients with an aIPI of 3-4 show a 5-year PFS of 40\% (Cheson et al. 1999). For younger patients with an aIPI of $1-4$, intensified protocols such as R-CHOEP, R-DA-EPOCH, or R-ACVB are applied resulting in a 5-year PFS of 50-60\%. High-dose chemotherapy (HD) with autologous hematopoietic stem cell transplantation (aHSCT) has been offered to younger DLBCL patients, but has not established itself as standard (Pfreundschuh et al. 2006).

Primary central nervous system lymphoma (PCNSL) is recognized as separate identity, and is currently treated with blood-brain-barrier passing protocols such as high-dose 
MTX, TT, and AraC followed by HD with aHSCT in younger patients (Ferreri et al. 2016). This approach results in a PFS of $60 \%$ as reported by several study groups (Illerhaus et al. 2012; Omuro et al. 2015; Cote et al. 2012; Hollender et al. 2000; Schorb et al. 2013). A particular adverse situation occurs when DLBCL patients present with both nodal disease and CNS involvement. This finding can be seen in less than $4 \%$ of primary diagnosis of DLBCL (Akkas and Vural 2013), and no standardized approach how to treat such patients has been developed. The retrospective outcome for this group of patients remains inferior with a 3-year survival of 26\% without, and 75\% with HD+aHSCT (Damaj et al. 2015).

There is few prospective data about the specific subgroup. An Italian study reported forty patients, including 16 PCL patients. After a combination therapy including HD-MTX, HD cytarabine, and HD etoposide combined with rituximab and liposomal cytarabine, it found a 5-year OS rate of $41 \%$ (Ferreri et al. 2015a). The MARIETTA trial reported 79 patients, among those 32 PCL patients, both relapsed and therapy naive. After three courses of MATRix (AraC, TT and R) as well as three courses of R-ICE (R, ifosfamide, cisplatin, E), intrathecal therapy, and BCNU/TT + aHSCT, a 2-year OS of $42 \%$ was reported (Ferreri et al. 2021).

We present the whole cohort of 16 patients who presented at our center with SCL between 2013 and 2018. They were treated uniformly with a newly developed protocol, which includes an induction with 4 cycles of R-CHOP-21 and R-MTX on day 14 ("Ping-Pong"), followed by dose intensification with 2 cycles R-TT/AraC and BCNU/TT followed by aHSCT. Feasibility, toxicity and outcomes are included in this report, demonstrating a high rate of disease control with an OS of $93.75 \%$ at a median of 4.8 years with manageable toxicity profile.

\section{Methods}

\section{Identification of patients}

Sixteen patients ( 8 male, 8 female) with a median age of 60.2 [34.7-76.7] years at diagnosis that were treated at our center between March 2013 and June 2018 were retrospectively analyzed for this study. All patients were discussed prior to commencing treatment in our NHL tumor board, consisting of a pathologist, a radiotherapist, a specialist in nuclear medicine, radiologists, and hematologists. Treatment was offered only to HD-eligible patients with $\mathrm{ECOG}<3$. An ECOG rating of 3 due to lymphoma was accepted. An ethical review committee approved retrospective analysis and data acquisition.

A standard operating procedure at our center requires that every newly diagnosed DLBCL is staged via full body computed tomography (CT)-scan, bone marrow biopsy, and verification of diagnosis via central pathology review. DLBCL were classified as recommended by the WHO classification of 2016 (Swerdlow et al. 2016). All patients with clinical signs of CNS involvement or IPI $>2$ received a cranial magnetic resonance tomography (MRI), and lumbar puncture with cerebrospinal fluid (CSF) and testing via cytology and flow cytometry. In addition, patients demonstrating potential dura involvement in CT scans were further evaluated via MRI of the involved region. Renal and hepatic assessment as well as HIV/Hepatitis diagnostics were performed in all subjects before chemotherapy. All patients had normal ejection fraction via echocardiography and normal lung function levels via spirometry.

Biopsy of the central nervous lymphoma manifestation was conducted in eight (50\%) of all patients enrolled. Another seven patients were enrolled after confirming infiltration via MRI, even if CSF analysis did not show meningeosis. One patient, although negative in MRI, presented with central Horner Syndrome and was clinically suspected to have diffuse cerebral lymphoma infiltration. In patients with HIV-associated DLBCL, antiviral therapy was continued. Response assessment was performed according to the Lugano classification (Cheson et al. 2014).

We retrospectively analyzed response after induction, consolidation, and HD, for toxicity and time-dependent outcome such as duration of response (DOR), progression-free survival (PFS), and overall survival (OS).

\section{Treatment protocol and response assessment}

Patients with high disease burden received a pre-phase of $100 \mathrm{mg}$ Prednisone for 5 days after staging completion. Then, the induction protocol of four times standard R-CHOP-21 (Pfreundschuh et al. 2006) with R-MTX 4 g/ $\mathrm{m}^{2}$ (Pfreundschuh et al. 2006) on day 14, followed by leucovorine rescue, was administered. Rituximab was given at $375 \mathrm{mg} / \mathrm{m}^{2}$ (Pfreundschuh et al. 2006) i.v. or $1400 \mathrm{mg}$ s.c. In patients with proven CSF involvement, intrathecal therapy was performed with liposomal cytarabine weekly (GarciaMarco et al. 2009) or triple therapy (MTX, AraC, and dexamethasone) three times a week additionally to systemic therapy. Intrathecal therapy was continued until no lymphoma cells were detected by both microscopic examination and flow cytometry.

Response assessment according to Lugano classification criteria (2008) was conducted 2-4 weeks after the last PingPong cycle via CT scan and MRI of the involved region (Cheson et al. 2014). Another bone marrow puncture was conducted before stem cell harvest if there had been infiltration at diagnosis.

If at least a partial response or better was documented, the patient was eligible for dose intensification including HD 
with aHSCT. To this end, two cycles of R-AraC/TT, repeated after 21 days, were conducted. $10 \mu \mathrm{g} / \mathrm{kg}$ body weight G-CSF were given to support autologous stem cell collection after the first cycle. Stem cell collection was initiated when leucocyte count was $>2500 / \mu \mathrm{l}$ and $\mathrm{CD} 34^{+}$count $>20 / \mathrm{nl}$. If those numbers could not be reached by G-CSF alone, we added Plerixafor at a daily dose of $0.24 \mathrm{mg} / \mathrm{kg}$ body weight. Harvest was considered sufficient when two transplants containing each $>2 \times 10$ (Cote et al. 2012) $\mathrm{CD} 34^{+}$cells $/ \mathrm{kg}$ body weight were collected. One week after harvest, another cycle of R-AraC/TT was conducted. A second response assessment was performed 2-3 weeks after initiation of the second cycle of R-AraC/TT via CT scan, MRI, and bone marrow puncture as described. We initiated HD 1 week after leukocyte regeneration. One transplant contained $>2 \times 10$ (Cote et al. 2012) CD $34^{+}$cells $/ \mathrm{kg}$ body weight. In Table 1, we give an overview of application mode and dosage; Fig. 1 shows the overall therapy regimen. A final response assessment was performed 4-6 weeks after transplantation via MRI/ CT or PET-CT scan.

\section{Supportive care and standard procedures}

Baseline procedures included daily laboratory tests and clinical evaluation during hospital accommodation. In outpatients, weekly controls of liver enzymes, renal function, and blood counts as well as a clinical evaluation were performed. All patients received prophylaxis with Aciclovir $800 \mathrm{mg} 2 \times /$ day and Cotrimoxazole or Pentacarinate inhalation during the treatment until 60 days after aHSCT. If the patient displayed mucositis grade 3 or higher, prophylaxis with oral

Table 1 Overview of chemotherapy regimen

\begin{tabular}{lll}
\hline R-CHOP & & \\
Rituximab i.v./s.c & $375 \mathrm{mg} / \mathrm{m}^{2} / 1400 \mathrm{mg}$ abs & Day 1 \\
$\begin{array}{l}\text { Cyclophosphamide } \\
\text { Doxorubicin }\end{array}$ & $750 \mathrm{mg} / \mathrm{m}^{2}$ i.v & Day 1 \\
Vincristin & $50 \mathrm{mg} / \mathrm{m}^{2}$ i.v & Day 1 \\
Prednisone & $2 / 1 \mathrm{mg}$ abs. i.v & Day 1 \\
R-MTX & $100 \mathrm{mg}$ abs. i.v & Days $1-5$ \\
Rituximab i.v./s.c & $375 \mathrm{mg} / \mathrm{m}^{2} / 1400 \mathrm{mg}$ abs & Day 0 \\
MTX & $4 \mathrm{~g} / \mathrm{m}^{2}$ & Day 1 \\
R-AraC/TT & & \\
Rituximab s.c & $1400 \mathrm{mg}$ abs. s.c & Day 1 \\
Cytarabine & $4 \mathrm{~g} / \mathrm{m}^{2}$ & Days $1-{ }^{\wedge} 2$ \\
Thiothepa & $40 \mathrm{mg} / \mathrm{m}^{2}$ & Day 2 \\
R-HD-BCNU/TT & & \\
Rituximab s.c & $1400 \mathrm{mg}$ abs & Day -7 \\
BCNU & $400 \mathrm{mg} / \mathrm{m}^{2}$ & Day -6 \\
Thiothepa & $2 \times 5 \mathrm{mg} / \mathrm{m}^{2}$ & Day $-5 /-4$ \\
aHSCT & $>2 \times 10^{6} \mathrm{CD} 34^{+}$cells/ kg & Day 0 \\
& body weight & \\
\hline
\end{tabular}

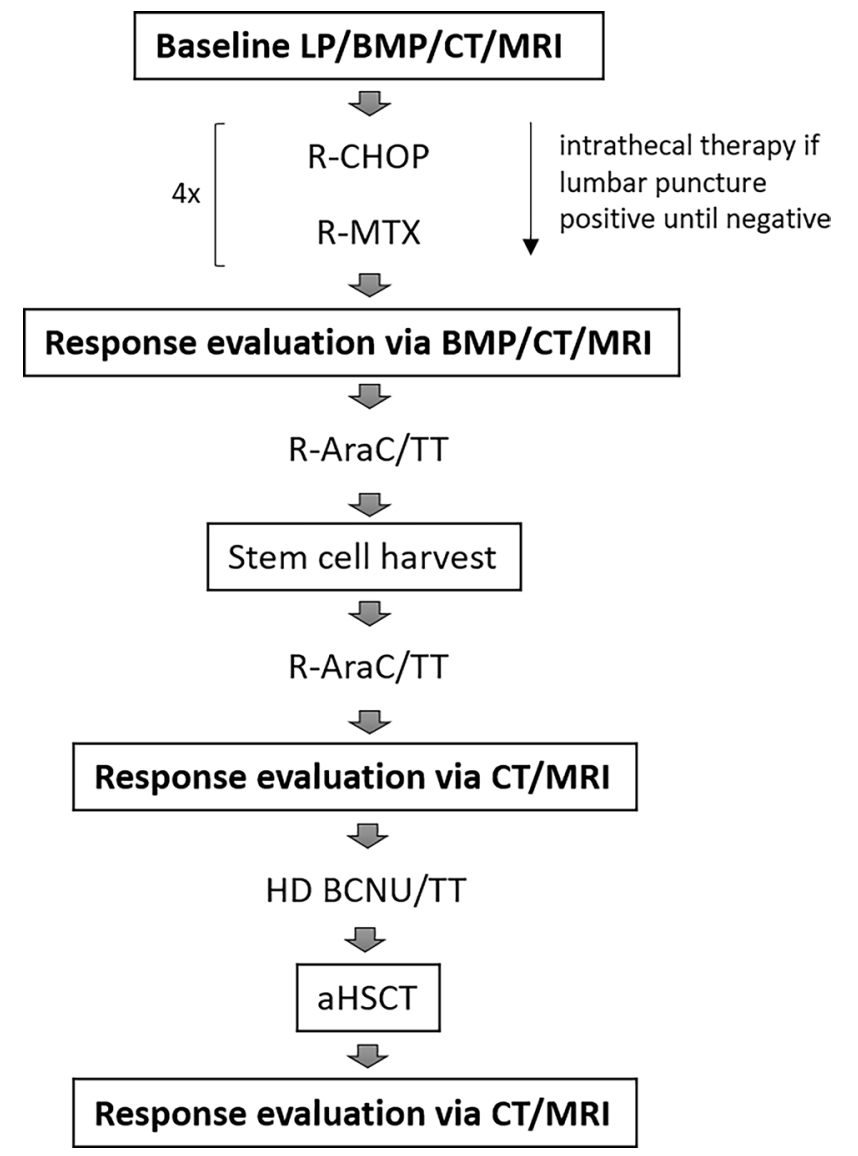

Fig. 1 Treatment and staging. $L P$ lumbar puncture, $B M P$ bone marrow puncture, $C T$ computed tomography, $C M R I$ cranial magnetic resonance imaging, $\mathrm{R}$-CHOP Rituximab, Cyclophosphamide, Vincristine, Prednisone, MTX methotrexate, AraC cytarabine, TT thiotepa, $H D$ high dose, $B C N U$ carmustine, aHSCT autologous hematopoietic stem cell transplantation

Amphotericine B and fluorchinolones was administered. Granulocyte colony-stimulating factor (G-CSF) was administered during periods with $<1000$ leukocytes/nl (Fig. 2).

\section{Adverse events reporting}

Toxicity data were retrospectively collected and graded based on the CTCAE 5.0 criteria. For collection, we analyzed all laboratory values before, during and after treatment, viewed all day-to-day clinical documentation and systematically consulted the patients about long-term effects and toxicities every 3-6 months. 


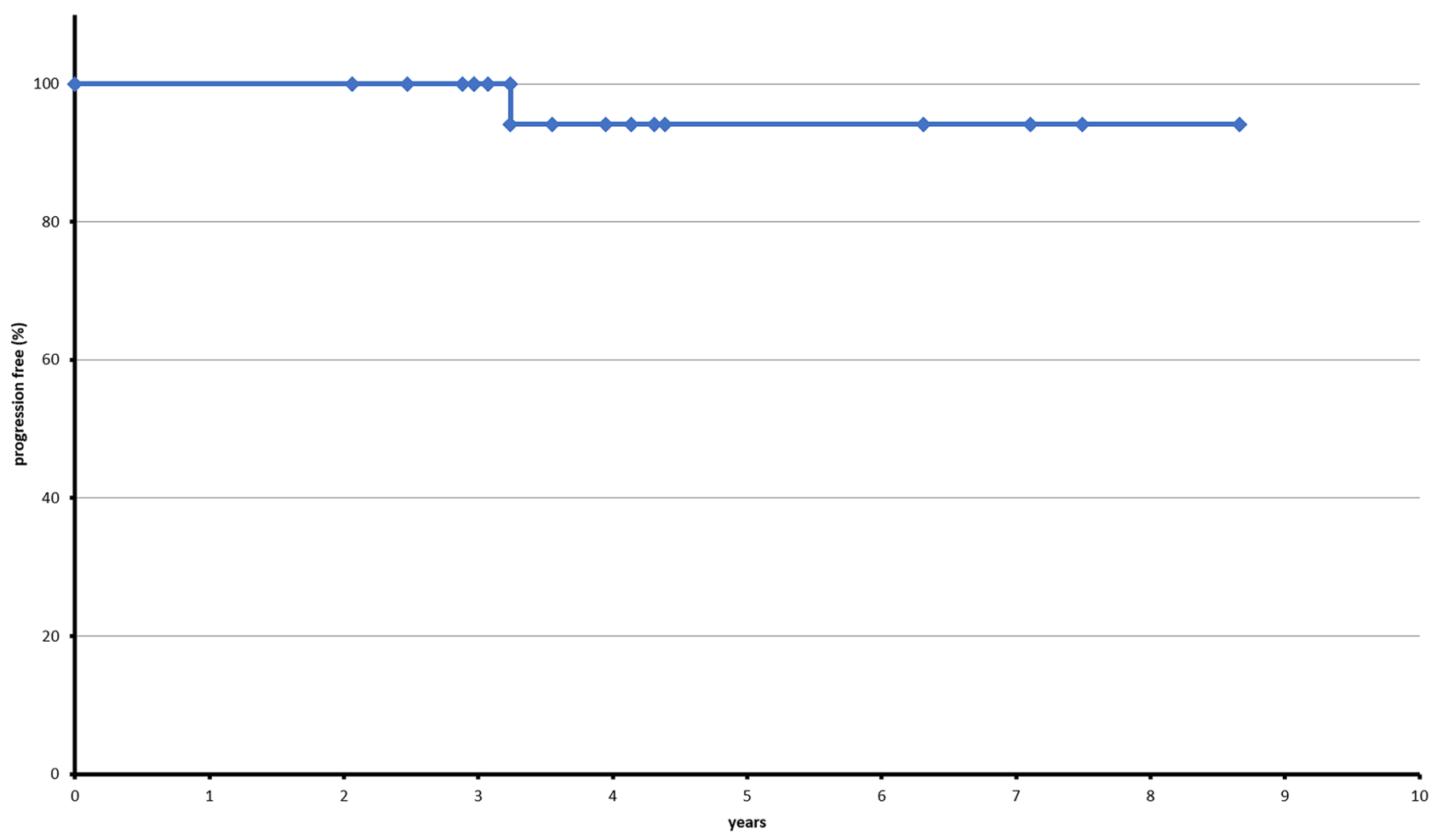

Fig. 2 PFS under study protocol. Median follow-up is 4.8 years, currently

\section{Results}

\section{Patient characteristics}

Overall, 16 patients with recently diagnosed PCL, treated between March 2013 and June 2018, are included in the analysis. Median age was 60.2 years, $50 \%$ of them were male and most patients had high-intermediate $(31.3 \%)$ or high-risk (37.5\%) lymphoma according to aggressive NHL IPI stratification. Three patients were graded ECOG 2. One patient was enrolled despite an ECOG 3 status, which was due to cerebral lymphoma. All other patients enrolled (75\%) had an ECOG performance status of 1. MYC status was negative where retrospective FISH analysis was possible. CNS involvement presented as cerebral infiltration in six patients, and spinal lymphoma infiltration was revealed in another six patients. In three patients, MRI revealed dura infiltration. One patient had a clinically evident central Horner's syndrome without possible MRI verification. Two patients had been irradiated on the right arm and duodenal bulb, respectively, with 40 Gy shortly before diagnosis of central lymphoma involvement. CSF lymphoma cells were detected in two patients, three patients did not undergo lumbar puncture at initial diagnosis. Patient characteristics are summarized in Table 2. Figure 3 shows central nervous infiltration types as diagnosed via MRI.
Toxicity and dose reduction of Ping-Pong protocol

All 16 patients received the first block of chemotherapy consisting of four cycles of R-CHOP and R-MTX (see Fig. 1). Age-adjusted Vincristine dose reduction was performed according to R-CHOP guidelines. In nine patients (56.3\%), no further dose reduction was required. All patients developed grade $3 / 4$ adverse events according to CTCAE. Acute kidney failure also occurred in $31.3 \%$ of the patients, all of which were considered MTX-associated. One patient received only $50 \%$ of the MTX dose after acute kidney failure during the second R-MTX cycle, and two patients discontinued MTX after acute renal failure or high-grade mucositis. Another patient suffered kidney failure, which remained a grade 3 chronic renal impairment. All other renal failures resolved without sequelae. None of the patients died due to treatmentrelated toxicity or complications. Grade 3 to 4 hematotoxicity was observed in all patients. On average, duration of the four cycles was 13.5 weeks, compared to a theoretical minimum of 12 weeks, suggesting good tolerability. Prolonged therapy duration was due to infections in all eight cases (50\%). During Ping-Pong, three of the patients required platelet transfusion, and 5 out of 16 received red blood cell transfusions. Significant liver enzyme elevations occurred in four cases (25\%) and were completely reversible. Severe complications included port infections in three cases (18.8\%) and Clostridium 


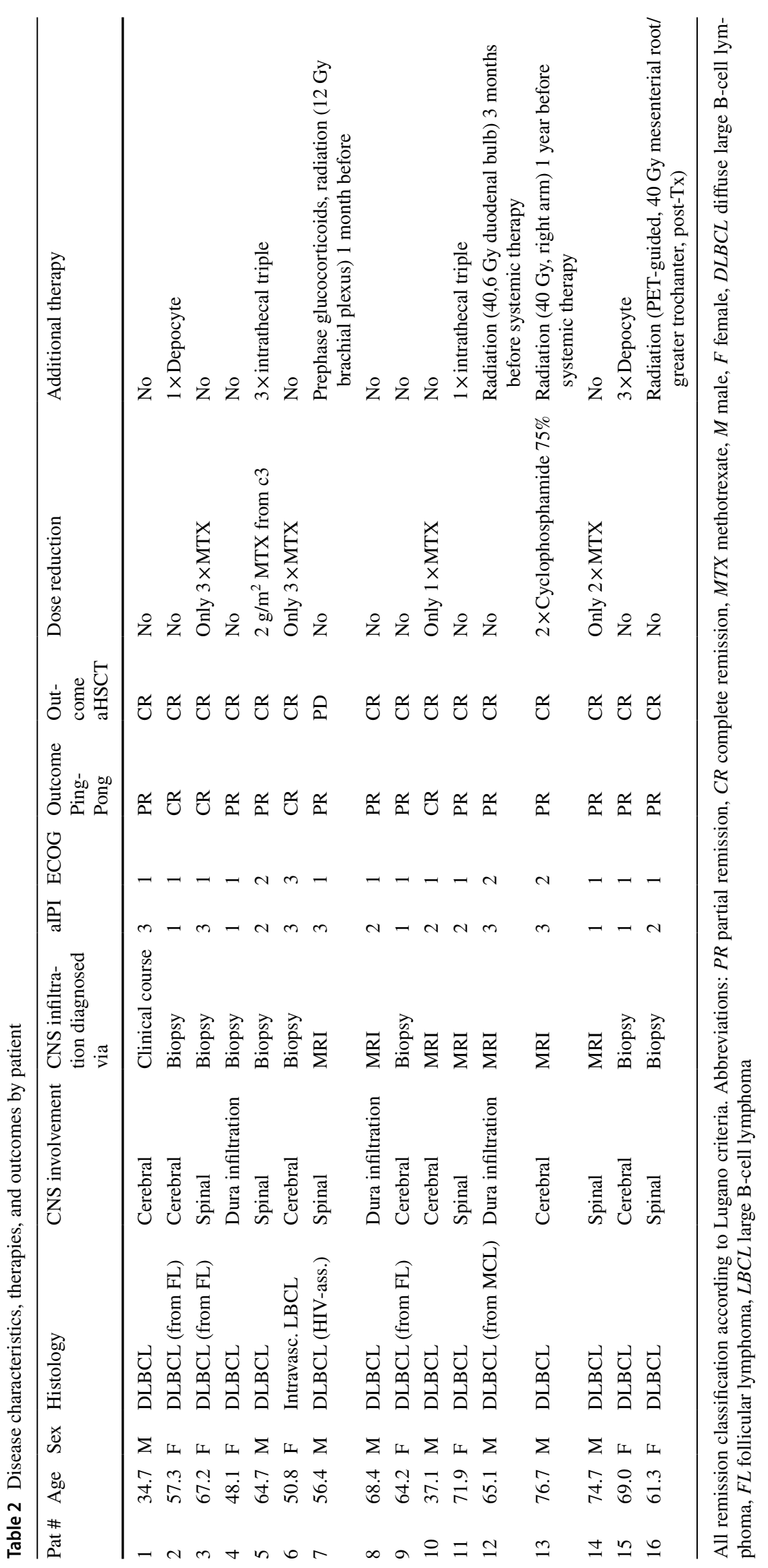


a

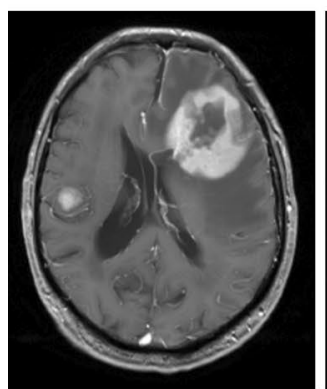

d

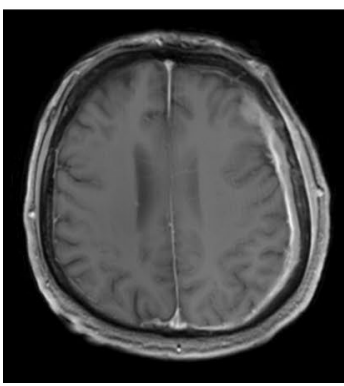

b

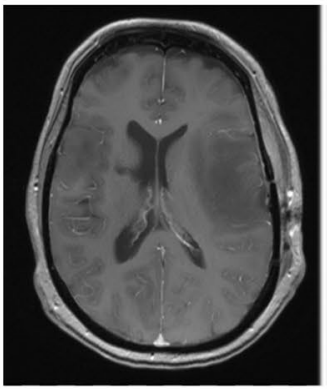

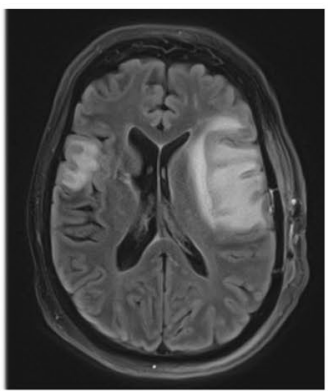

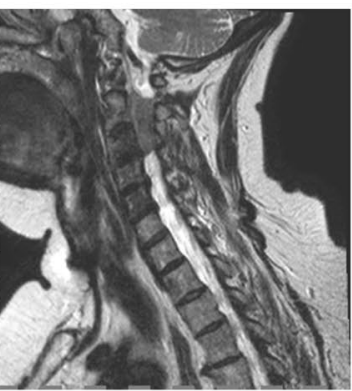

$f$
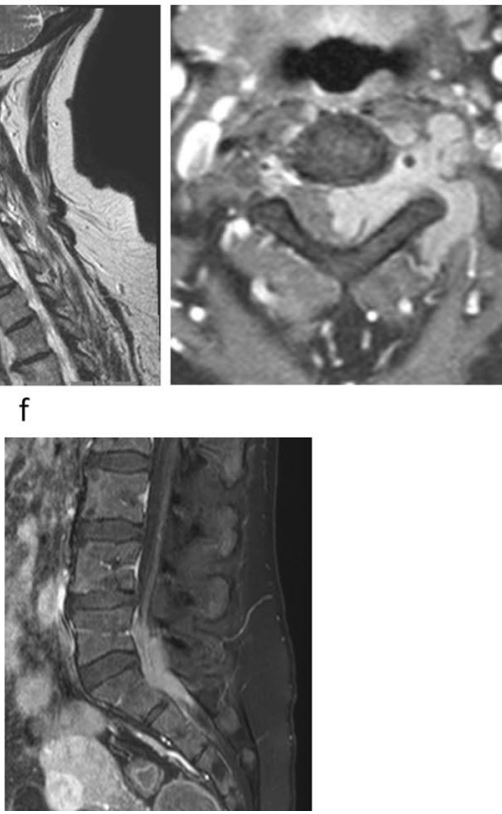

Fig. 3 Radiologic aspects of central lymphoma manifestations in our study. a Cerebral DLBCL. T1-weighted (T1-w) 2D FLASH sequence post-contrast. b Intravascular LBCL of small- and medium-sized intracerebral and meningeal vessels. T1-weighted gradient-recalled echo (GRE) post-contrast and T2 FLAIR TSE BLADE sequence. c Spinal dura infiltration of DLBCL. T2-weighted turbo spin-echo
(TSE). T1 fat saturated turbo spin-echo (TSE) post-contrast. d Cerebral dura infiltration of DLBCL. T1-weighted (T1-w) 2D FLASH sequence post-contrast. e Bone-associated lymphoma with cerebral infiltration. T1-weighted (T1-w) Turbo Spin-Echo (TSE) sequence post-contrast. f Spinal dura infiltration of DLBCL. Fat-saturated T1-weighted (T1-w) Turbo Spin-Echo (TSE) sequence post-contrast
Table 3 Grade 3/4

complications during R-CHOP/

R-MTX and AraC/TT and HD

with aHSCT (CTCAE)

\begin{tabular}{lll}
\hline Complication & During Ping-Pong & During AraC/TT + HD \\
\hline Acute kidney failure (via RIFLE criteria) & $5(31.3 \%)$ & 0 \\
Grade 3/4 anemia & $5(31.3 \%)$ & $11(73.3 \%)$ \\
Grade 3/4 thrombopenia & $4(25.0 \%)$ & $14(93.3 \%)$ \\
Febrile neutropenia & $4(25.0 \%)$ & $7(46.7 \%)$ \\
Elevated liver enzymes & $4(25.0 \%)$ & $2(13.3 \%)$ \\
Port infection & $3(18.8 \%)$ & 0 \\
Clostridium difficile-associated diarrhea & $2(12.5 \%)$ & $1(6.7 \%)$ \\
Mucositis & $2(12.5 \%)$ & 0 \\
Urinary tract infection & $2(12.5 \%)$ & $1(6.7 \%)$ \\
EBV/CMV/HSV reactivation & $1(6.3 \%)$ & 0 \\
Candida infection & $1(6.3 \%)$ & 0 \\
Delirium & 0 & $2(13.3 \%)$ \\
Sepsis & 0 & $1(6.7 \%)$ \\
\hline
\end{tabular}

$E B V$ Epstein-Barr Virus, $C M V$ cytomegaly virus, $H S V$ herpes simplex virus difficile-associated diarrhea in two cases. Table 3 presents an overview of grades 3-4 complications. No deaths occurred.

All patients qualified for the intensification with two cycles of R-AraC/TT with stem cell harvest after the first cycle and HD-BCNU/TT followed by aHSCT, a regimen often used in primary CNS lymphoma (see Fig. 1). One patient received radiation (12 Gy) of the brachial plexus due to lymphoma-associated sensomotoric impairment during this block of therapy. 


\section{Response after Ping-Pong protocol}

We used a combination of CT scan and MRI of the involved region to evaluate response in all 16 patients enrolled after four cycles R-CHOP/R-MTX.

According to IWG 2007 response criteria, all patients achieved a response, resulting in an ORR of $100 \%$. This includes 4 (25\%) complete remissions (CR) and 11 partial remissions $(75 \%)$. We observed no stable or progressive disease under the Ping-Pong protocol.

\section{Treatment-emergent adverse events of AraC/TT and high-dose chemotherapy}

15 of the patients included proceeded to AraC/TT and subsequent HD with aHSCT (see Fig. 1) consisting of 2 cycles AraC/TT and HD-BCNU/TT.

This block was applied during a medium of 10, 6 weeks, compared to a minimum duration of 10 weeks, suggesting good tolerability. At least $4 \times 10^{6} / \mathrm{kg} \mathrm{CD} 34^{+}$cells for each of the two transplants were collected after the first cycle of R-AraC/TT. However, three patients (18.75\%) required Plerixafor application to mobilize a sufficient number of stem cells.

14 patients $(93.3 \%)$ required platelet transfusions, and $11(73.3 \%)$ red blood cell transfusions after HD. In seven, febrile neutropenia occurred, which proved well manageable. Severe complications included medication-associated deliria in two cases (13.3\%) and sepsis with ICU transfer in one case (6.7\%). No dose reductions were necessary. All patients were able to complete the protocol and qualified for AraC/TT and HD with aHSCT. None of the patients died due to treatment-related toxicity or complications. Grades 3-4 hematotoxicity was observed in all patients. 13 of the patients developed infectious complications grade 3 or higher. An overview of grades 3-4 complications is given in Table 3. Median time to neutrophil regeneration was 8.2 days.

\section{Response after successive AraC/TT and high-dose chemotherapy protocol}

Ping-Pong was conducted to prepare the enrolled patients for the local standard of care protocol including two cycles of R-AraC/TT with stem cell harvest after the first cycle and HD-BCNU/TT followed by aHSCT (see Fig. 1). 15 patients (93.75\%) reached CR in cMRI and thoracoabdominal CT scan combined. One patient $(6.25 \%)$ showed signs of progression during the first cycle of R-AraC/TT after response to Ping-Pong protocol and was not continued, showing a PFS of 4 months. Histologically, he suffered from HIV-related lymphoma which has historically been linked to worse prognosis and therapy outcome (Diamond et al. 2006).

Table 2 outlines all therapy modifications made. All patients who had undergone aHSCT achieved lasting CR. Although median PFS and OS survival have not been reached after 4.8 years. So far, we have not experienced relapses. One patient required PET-guided consolidating radiation $(40 \mathrm{~Gy})$ of the trochanter region.

\section{PFS, DOR, and overall survival}

Current median follow-up is 4.8 years (see Fig. 2). So far, no further relapses were observed. The overall survival rate is at $93.25 \%$, currently. Correspondingly, overall survival for transplanted patients is at $100 \%$. Follow-up visits take place every 3 months for the first 3 years, then every 6 months until 5 years after HD.

\section{Long-term toxicities}

During follow-up after therapy completion, patients were systematically interviewed about long-term effects every 3-6 months. Most commonly, patients complained about fatigue (40\%). Other problems reported were polyneuropathy and cognitive impairment. Three patients reported persistence of problems caused by irreversible damage caused by lymphoma manifestations. These include paraneoplastic stiff man syndrome, blindness, and back pain, each $6.7 \%$, respectively. One patient suffered chronic renal impairment and heart failure, and hematopoietic recovery was incomplete in another patient. Six patients did not report any sequelae. None of the patients received further therapy or developed a secondary malignancy to this point.

\section{Discussion}

Central nervous involvement is a rare and adverse presentation in lymphoma, offering significantly worse outcomes. Various approaches exist to optimize therapy regimen. In 2015, the LYSA and LOC network published results of a retrospective 60-case study on heterogeneous up-front treatments for SCL. Here, an overall progression-free 3-yearsurvival of $40-50 \%$ was reported after HD with aHSCT, suggesting an important role for HD consolidation therapy in this subset of patients (Damaj et al. 2015). However, Wight et al. did not observe a statistically significant improvement in 2-year PFS after various induction protocols followed by HD compared to no HD (Wight et al. 2019). Current protocols for primary central nervous lymphoma and relapsed synchronous systemic and central nervous lymphoma offer similar outcomes after HD with aHSCT (El-Galaly et al. 
2018; Kasenda et al. 2012). Neither of the studies provide data about complications or tolerability.

Two prospective trials studied a similar patient cohort with concomitant systemic and central nervous lymphoma. In 2015, Ferreri et al. reported a 5-year OS rate of $41 \%$ with combination therapy including HD-MTX, HD cytarabine, and HD etoposide combined with rituximab and liposomal cytarabine followed by BCNU/TT consolidation and aHSCT. The study included 16 PCL patients, but does not provide data about the specific subgroup (Ferreri et al. 2015a).

The MARIETTA trial reported 32 patients PCL among a greater collective, both relapsed and therapy naive. After three courses of MATRix (AraC, TT and R) as well as three courses of R-ICE (R, ifosfamide, cisplatin, E), intrathecal therapy, and BCNU/TT + aHSCT, a 2-year OS of $42 \%$ was reported (Ferreri et al. 2021).

The idea to combine HD-MTX with common therapies for systemic lymphoma is not new; Chihara et al. reported results for EPOCH-R (etoposide, prednisone, vincristine, cyclophosphamide, doxorubicin, and R) with mid-cycle HD-MTX. After a median of five cycles, all of the eight patients with PCL achieved CR with an OS of $100 \%$ at a median follow-up at 11 months. Four of the patients received consolidating aHSCT (Chihara et al. 2017). Details on toxicities are not given.

This supports our findings, suggesting high effectivity of mid-cycle MTX in PCL and a significant effect of CNSintensive induction therapy towards acceptable OS results in this challenging patient population. Puckrin et al. recently presented retrospective data on a large cohort of patients with high IPI lymphoma. Here, prophylactic HD-MTX did not reduce the number of CNS relapses, questioning the role for HD-MTX in long-term PFS (Puckrin et al. 2020). However, MTX is considered a backbone for patients with central lymphoma.

In other retrospective studies, several groups reported significantly worse OS rates of roughly $50 \%$ in heterogeneously treated cohorts, mostly without HD + aHSCT without giving details on toxicities (Wight et al. 2019; Maciocia et al. 2016; Nijland et al. 2017).

The worse outcomes reported by other groups can be due to the significantly worse performance status in their cohorts. Often, patients did not qualify for HD, which we believe to be a crucial part of successful long-term therapy. Consolidating HD is associated with a lower risk of CNS relapse in high-risk lymphoma (Puckrin et al. 2020). For the prevention of secondary central nervous lymphoma, lack of cerebral low-dose intensity of CNS-effective treatment is a long-known risk factor, and HD with aHSCT has been recommended (Bromberg et al. 2013; Doolittle et al. 2008; Maziarz et al. 2013).

We provide further evidence that consolidating $\mathrm{HD}$ with aHSCT, if tolerated by the patient, should be pursued in PCL patients to optimize outcomes. However, we report better OS data than other retrospective reports. Thus, selection biases must be thoroughly discussed.

Therapy outcomes in lymphoma greatly depend on risk stratification. All patients enrolled presented an Ann-Arbor stage IV and had a high-intermediate to high-risk classification according to IPI, suggesting a high-risk patient cohort. However, our protocol is more aggressive than the uniformly applied R-CHOP, and other approaches such as prophylactic HD-MTX did not reduce the number of CNS relapses (Puckrin et al. 2020). This may overcome the impact of high IPI for the prognosis of our cohort.

In addition, none of the 14 analyzed patients had a double or triple hit lymphoma. While the CNS involvement in our cohort comes with high-risk classification, the lack of molecular high-risk patients may constitute a favorable population within this range. Interestingly, although three of the DLBCL were histopathologically suspected to have developed from follicular lymphoma (FL), no MYC alterations were detected via FISH. However, DLBCL transformation from FL can be attributed to other mutations we did not screen for (Fischer et al. 2018).

Only in 8 out of 16 patients, CNS lymphoma infiltration was histologically confirmed as biopsy often proved not feasible, too risky, or without therapeutic consequence. The good outcome in our cohort may be influenced by overtreatment. This may especially be true for the three patients that were enrolled due to lymphoma infiltration of the dura mater. One patient, although MRI-negative, was clinically suspected to have diffuse cerebral lymphoma infiltration, but the initial Horner's syndrome gradually recovered under therapy, suggesting success of CNS-directed therapy. HD is known to reduce the number of relapses in patients with high aIPI (Puckrin et al. 2020), which may solely explain the lack of long-term relapses in our cohort.

This study was performed at our center only. The possible confounding factors limit the value of the data presented.

Notably, other reports included a significantly higher percentage of patients had a performance status of three or higher, which may partly explain the difference in outcome. While our cohort offers far better OS data, it is a retrospective analysis and thus susceptible to selection biases. All patients that received Ping-Pong at our center are included in this study.

Concerning adverse events during induction therapy, only few studies offer detailed information for this specific patient subset. Ferreri et al. reported better tolerability data for the SCNSL1 trial, with mainly hematologic high-grade and rare infectious complications, whereas only $75 \%$ of all patients suffered high-grade complications (Ferreri et al. 2015b).

During the MARIETTA trial, SAEs occurred in slightly over $50 \%$ of the patients, with hematologic events being the most common (Ferreri et al. 2021). However, only 53\% 
qualified for planned HD and aHSCT. Four therapy-associated deaths occurred in both trials.

Maciocia et al. reported in individualized consolidation therapy regimens with and without HD and aHSCT after 1-4 cycles R-IDARAM significantly less-grade 3/4 neutropenia, anemia, and thrombocytopenia (55, 62, and $12 \%$, retrospectively). Hepatotoxicity occurred in $14 \%$ of cycles (Maciocia et al. 2016).

However, we observed grade $3 / 4$ toxicities in all patients. Notably, the underestimation of side effects is a major limitation of our retrospective study and should be considered when comparing data, suggesting an even higher toxicity of Ping-Pong with HD consolidation, and may point to a more aggressive regimen at our center towards a relatively healthy population. No therapy-associated deaths occurred during our protocol, which may solely be due to the low number of patients treated.

Complication rates during AraC/TT and stem cell harvest after Ping-Pong are similar to current high-dose concepts of central nervous lymphoma (Illerhaus et al. 2006). Average duration of aplasia during HD was comparable at 8.5 days, suggesting adequate dosing and usual aHSCT complication patterns. In all patients, stem cell mobilization was successful. However, a significant number required Plerixafor application to mobilize a sufficient number of stem cells. The relatively high dosage of chemotherapy compared to other concepts before stem cell harvest may play a role here. Other groups reported no mobilization issues after HD-MTX and R-AraC/TT in PCNSL patients (Kasenda et al. 2012; Cheng et al. 2003).

This study provides a promising approach to the rare and difficult-to-treat entity of systemic lymphoma with primary CNS involvement. At our center, R-CHOP and HD-MTX with HD and aHSCT were feasible. Our data suggest effectiveness for transplant-eligible patients with concomitant peripheral and central nervous B-cell lymphoma. Provided the low incidence, not many patients were eligible for this study. Protocol application will proceed, given these promising preliminary results.

\begin{abstract}
Author contributions MJS collected the data, contributed data, performed analysis and wrote the paperas principal investigator. FCK contributed data. AR contributed data. EG-H contributed data. AH contributed data. HE conceived theanalysis. MST conceived and designed the analysis and wrote the paper. JD conceived and designed the analysis, collected data and wrote the paperas senior author.
\end{abstract}

Funding Open Access funding enabled and organized by Projekt DEAL.

Data availability Thedata that support the findings of this study are available from thecorresponding author upon request. The authors declare that all data supportingthe findings of this study are available within the paper.

\section{Declarations}

Conflict of interest The authors report no competing interests.

Open Access This article is licensed under a Creative Commons Attribution 4.0 International License, which permits use, sharing, adaptation, distribution and reproduction in any medium or format, as long as you give appropriate credit to the original author(s) and the source, provide a link to the Creative Commons licence, and indicate if changes were made. The images or other third party material in this article are included in the article's Creative Commons licence, unless indicated otherwise in a credit line to the material. If material is not included in the article's Creative Commons licence and your intended use is not permitted by statutory regulation or exceeds the permitted use, you will need to obtain permission directly from the copyright holder. To view a copy of this licence, visit http://creativecommons.org/licenses/by/4.0/.

\section{References}

Akkas BE, Vural GU (2013) The incidence of secondary central nervous system involvement in patients with non-Hodgkin's lymphoma as detected by 18F-FDG PET/CT. Nucl Med Commun 34(1):50-56

Bromberg JE, Doorduijn JK, Illerhaus G, Jahnke K, Korfel A, Fischer L et al (2013) Central nervous system recurrence of systemic lymphoma in the era of stem cell transplantation-an International Primary Central Nervous System Lymphoma Study Group project. Haematologica 98(5):808-813

Cheng T, Forsyth P, Chaudhry A, Morris D, Glück S, Russell J et al (2003) High-dose thiotepa, busulfan, cyclophosphamide and ASCT without whole-brain radiotherapy for poor prognosis primary CNS lymphoma. Bone Marrow Transpl 31(8):679-685

Cheson BD, Horning SJ, Coiffier B, Shipp MA, Fisher RI, Connors JM et al (1999) Report of an international workshop to standardize response criteria for non-Hodgkin's lymphomas. J Clin Oncol 17(4):1244-1244

Cheson BD, Fisher RI, Barrington SF, Cavalli F, Schwartz LH, Zucca E et al (2014) Recommendations for initial evaluation, staging, and response assessment of Hodgkin and non-Hodgkin lymphoma: the Lugano classification. J Clin Oncol 32(27):3059

Chihara D, Fowler NH, Oki Y, Fanale MA, Fayad LE, Westin JR et al (2017) Dose-adjusted EPOCH-R and mid-cycle high dose methotrexate for patients with systemic lymphoma and secondary CNS involvement. Br J Haematol 179(5):851-854

Cote GM, Hochberg EP, Muzikansky A, Hochberg FH, Drappatz J, McAfee SL et al (2012) Autologous stem cell transplantation with thiotepa, busulfan, and cyclophosphamide (TBC) conditioning in patients with CNS involvement by non-Hodgkin lymphoma. Biol Blood Marrow Transpl 18(1):76-83

Damaj G, Ivanoff S, Coso D, Ysaebert L, Choquet S, Houillier C et al (2015) Concomitant systemic and central nervous system nonHodgkin lymphoma: the role of consolidation in terms of high dose therapy and autologous stem cell transplantation. A 60-case retrospective study from LYSA and the LOC network. Haematologica 100(9):1199-1206

Diamond C, Taylor TH, Im T, Anton-Culver HJL (2006) Presentation and outcomes of systemic non-Hodgkin's lymphoma: a comparison between patients with acquired immunodeficiency syndrome (AIDS) treated with highly active antiretroviral therapy and patients without AIDS. Leuk Lymphoma 47(9):1822-1829

Doolittle ND, Abrey LE, Shenkier TN, Tali S, Bromberg JE, Neuwelt EA et al (2008) Brain parenchyma involvement as isolated central nervous system relapse of systemic non-Hodgkin lymphoma: 
an International Primary CNS Lymphoma Collaborative Group report. Blood 111(3):1085-1093

El-Galaly TC, Cheah CY, Bendtsen MD, Nowakowski GS, Kansara $\mathrm{R}$, Savage KJ et al (2018) Treatment strategies, outcomes and prognostic factors in 291 patients with secondary CNS involvement by diffuse large B-cell lymphoma. Eur J Cancer 93(1):57-68

Ferreri AJ, Donadoni G, Cabras MG, Patti C, Mian M, Zambello R et al (2015a) High doses of antimetabolites followed by highdose sequential chemoimmunotherapy and autologous stem-cell transplantation in patients with systemic B-cell lymphoma and secondary CNS involvement: final results of a multicenter phase II trial. J Clin Oncol 33(33):3903-3910

Ferreri AJ, Donadoni G, Cabras MG, Patti C, Mian M, Zambello R et al (2015b) High doses of antimetabolites followed by highdose sequential chemoimmunotherapy and autologous stem-cell transplantation in patients with systemic B-cell lymphoma and secondary CNS involvement: final results of a multicenter phase II trial. J Clin Oncol 33(33):3903-3910

Ferreri AJ, Cwynarski K, Pulczynski E, Ponzoni M, Deckert M, Politi LS et al (2016) Chemoimmunotherapy with methotrexate, cytarabine, thiotepa, and rituximab (MATRix regimen) in patients with primary CNS lymphoma: results of the first randomisation of the International Extranodal Lymphoma Study Group-32 (IELSG32) phase 2 trial. Lancet Haematol 3(5):e217-e227

Ferreri AJ, Doorduijn JK, Cattaneo C, Cabras MG, Smith J, Ilariucci F et al (2021) MATRix-RICE therapy and autologous haematopoietic stem-cell transplantation in diffuse large B-cell lymphoma with secondary CNS involvement (MARIETTA): an international, single-arm, phase 2 trial. Lancet Haematol 2(8):e110-e121

Fischer T, Zing NPC, Chiattone CS, Federico M, Luminari S (2018) Transformed follicular lymphoma. Ann Hematol 97(1):17-29

Garcia-Marco JA, Panizo C, Garcia ES, Deben G, Alvarez-Larran A, Barca EG et al (2009) Efficacy and safety of liposomal cytarabine in lymphoma patients with central nervous system involvement from lymphoma. Cancer 115(9):1892-1898

Hollender A, Kvaloy S, Lote K, Nome O, Holte H (2000) Prognostic factors in 140 adult patients with non-Hodgkin's lymphoma with systemic central nervous system (CNS) involvement. A single centre analysis. Eur J Cancer 36(14):1762-1768

Illerhaus GMR, Ihorst G, Guttenberger R, Ostertag C, Derigs G, Frickhofen N, Feuerhake F, Volk B, Finke J (2006) High-dose chemotherapy with autologous stem-cell transplantation and hyperfractionated radiotherapy as first-line treatment of primary CNS lymphoma. J Clin Oncol 24:3865-3870

Illerhaus G, Fritsch K, Egerer G, Lamprecht M, von Bubnoff N, Wolf $\mathrm{H}-\mathrm{H}$ et al (2012) Sequential high dose immuno-chemotherapy followed by autologous peripheral blood stem cell transplantation for patients with untreated primary central nervous system lymphoma-a multicentre study by the Collaborative PCNSL Study Group Freiburg. Blood 120(121):302
Kasenda B, Schorb E, Fritsch K, Finke J, Illerhaus G (2012) Prognosis after high-dose chemotherapy followed by autologous stem-cell transplantation as first-line treatment in primary CNS lymphoma-a long-term follow-up study. Ann Oncol 23(10):2670-2675

Maciocia P, Badat M, Cheesman S, D'Sa S, Joshi R, Lambert J et al (2016) Treatment of diffuse large B-cell lymphoma with secondary central nervous system involvement: encouraging efficacy using CNS-penetrating R-IDARAM chemotherapy. Br J Haematol 172(4):545-553

Maziarz RT, Wang Z, Zhang MJ, Bolwell BJ, Chen AI, Fenske TS et al (2013) Autologous haematopoietic cell transplantation for non-H odgkin lymphoma with secondary CNS involvement. Br J Haematol 162(5):648-656

Nijland M, Jansen A, Doorduijn JK, Enting RH, Bromberg JE, KluinNelemans HC (2017) Treatment of initial parenchymal central nervous system involvement in systemic aggressive B-cell lymphoma. Leuk Lymphoma 58(9):2074-2079

Omuro A, Correa DD, DeAngelis LM, Moskowitz CH, Matasar MJ, Kaley TJ et al (2015) R-MPV followed by high-dose chemotherapy with TBC and autologous stem-cell transplant for newly diagnosed primary CNS lymphoma. Blood 125(9):1403-1410

Pfreundschuh M, Trümper L, Österborg A, Pettengell R, Trneny M, Imrie $\mathrm{K}$ et al (2006) CHOP-like chemotherapy plus rituximab versus CHOP-like chemotherapy alone in young patients with goodprognosis diffuse large-B-cell lymphoma: a randomised controlled trial by the MabThera International Trial (MInT) Group. Lancet Oncol 7(5):379-391

Puckrin R, El Darsa H, Ghosh S, Peters A, Stewart DA (2020) Lack of effectiveness of intravenous high-dose methotrexate for prevention of CNS relapse in patients with high-risk DLBCL: a retrospective analysis from Alberta, Canada. Blood 136:26-27

Schorb E, Kasenda B, Atta J, Kaun S, Morgner A, Hess G et al (2013) Prognosis of patients with primary central nervous system lymphoma after high-dose chemotherapy followed by autologous stem cell transplantation. Haematologica 98(5):765-770

Swerdlow SH, Campo E, Pileri SA, Harris NL, Stein H, Siebert R et al (2016) The 2016 revision of the World Health Organization classification of lymphoid neoplasms. Blood 127(20):2375-2390

Wight JC, Yue M, Keane C, Johnston A, Linton K, Chin C et al (2019) Outcomes of synchronous systemic and central nervous system (CNS) involvement of diffuse large B-cell lymphoma are dictated by the CNS disease: a collaborative study of the Australasian Lymphoma Alliance. Br J Haematol 187(2):174-184

Publisher's Note Springer Nature remains neutral with regard to jurisdictional claims in published maps and institutional affiliations. 